\title{
Evaluation of Prefix Delegation-Based Route Optimization Schemes for NEMO
}

\author{
Abu Zafar M. Shahriar and Mohammed Atiquzzaman \\ School of Computer Science \\ University of Oklahoma, OK73019, USA \\ Email: \{shahriar, atiq\}@ou.edu
}

\begin{abstract}
Among the schemes, proposed in the literature to solve the route optimization problem in NEtwork MObility (NEMO), the prefix delegation-based schemes perform better than other schemes. Depending on the way the prefix are delegated, the prefix delegation-based schemes result in difference in performance under different speed at various distance from the home network (network to which mobile network usually belongs). Therefore, there is a need to evaluate the performance of individual prefix delegation-based scheme to find an appropriate scheme based on speed and distance from home network. In this paper, we identify the differences of the prefix delegation-based schemes using simulation. Results reveal that performance of the schemes depends on speed of the network and distance from home network.
\end{abstract}

\section{INTRODUCTION}

Internet Protocol (IP), being tied to geographical location, does not allow a host to move between networks with continuity of connections at upper layers, and retaining reachabiity. A host, moving from one subnetwork to another, must acquire a new address resulting in loss of connections and reachability. To support host mobility, IETF designed Mobile IPv4 (MIPv4) [1] and Mobile IPv6 (MIPv6) [2] that let hosts to obtain new address, employ mobility management entities, and use signaling for location tracking.

Host mobility support is inefficient for managing mobility of a group of hosts, moving together, such as hosts in a vehicle. To overcome the inefficiency that arises from signaling of all hosts, IETF proposed NEtwork MObility (NEMO), and designed a Basic Support Protocol (BSP) [3]. In NEMO, hosts are in a subnetwork called mobile network with one or more mobile routers that manage mobility on behalf of all the hosts. A mobile network, attached to another mobile network, forms a nested mobile network. Nesting level, having an important consequence on performance of NEMO BSP, can be multiple when mobile networks form a hierarchy.

In NEMO BSP, communication with the mobile network is done through a bidirectional tunnel between the mobile router and its home agent resulting in a suboptimal route incurring the problems of higher header overhead and end-toend delay [4]. In MIPv6, the problems were addressed using route optimization (achieved by the mobile host inform its current location to the communicating host) which is difficult to achieve in NEMO because hosts inside the mobile network are unaware of their location. Moreover, nesting results in

The work has been supported by NASA Grant NNX06AE44G. communication through multiple home agents using multiple tunnels to intensify the problems. Therefore, most of the research efforts in NEMO concentrate on route optimization.

The schemes [5] [6], proposed in the literature, take various approaches for route optimization by avoiding packets going through home agents. Lim et al. [6] classified the schemes based on the approaches used i.e. recursive approach, hierarchical approach and prefix delegation- based approach (called aggregate \& surrogate approach in [6]). Approaches cost performance metrics such as memory consumption, signaling, processing at mobility entities (home agents and mobile routers) and handoff delay to different extents. Since prefix delegation-based approach performs better than other approaches [6], we concentrate to analyze further the performance of the prefix delegation-based schemes.

Prefix delegation-based schemes differ in address obtaining procedure resulting in differences in handoff delay that reduces throughput. Consequently, throughput of the schemes varies with handoff frequency which depends mobility speed. In addition, schemes differ in optimizing route of some mobile network hosts yielding different end-to-end delay (and hence, throughput) when mobile network is away (i.e. end-to-end delay between the home agent and the mobile network increases) from home agent. Therefore, throughput characteristics of the schemes are not obvious under various mobility speed of a mobile network while at different end-to-end delay from home agent. Our goal is to compare the throughput of the prefix delegation-based schemes at different speeds and end-to-end delays from the home agent.

To compare, we selected three schemes - Simple Prefix Delegation (SPD) [7], Mobile IPv6 based Route Optimization (MIRON) [8] and Optimal Path Registration (OPR) [9] that are representative of all prefix delegation-based schemes considering the differences mentioned in the previous paragraph. No comparison of these schemes is available in the literature. Moreover, performance evaluation methods of the schemes in the literature in which they were proposed are different (i.e. simulation or analytical or testbed) resulting in inability to compare the schemes. We simulate all the schemes to perform an even comparison.

Our contributions are: (i) Identification of the differences among the schemes, and (ii) comparison of throughput of the schemes under various speeds of the mobile network at different end-to-end delay from the home agent. Simulation 
results show that speed of mobility and the end-to-end delay between the mobile network and the home agent determine performance superiority of MIRON and SPD, and do not affect throughput of OPR. In addition to simulation-based comparison, we comment on suitability of implementation of the schemes. Results of this research will help network designers to select an appropriate route optimization scheme based on mobility characteristics and distance of the mobile network from the home agent.

The rest of the paper is organized as follows. Sec. II summarizes NEMO followed by an overview of the selected route optimization schemes and their differences in Sec. III. Details of simulation and results are discussed in Sec. IV followed by concluding remarks in Sec. V.

\section{NEMO}

In this section, we briefly summerize NEMO architecture and NEMO BSP that will help to understand the prefix delegation based route optimization schemes presented in Sec. III.

\section{A. NEMO Architecture}

Fig. 1 shows the architecture of a mobile network to manage the mobility of the nodes as a network [3]. One or more routers called Mobile Router (MR) are employed to act as gateways for the nodes in the network. There could be different types of nodes inside the mobile network each called a Mobile Network Node (MNN). Different types of MNNs are - Local Fixed Node (LFN) that does not move with respect to the mobile network, Local Mobile Node (LMN) that usually resides in the mobile network and can move to other networks, Visiting Mobile Node (VMN) that get attached to the mobile network from another network, and MR that can be an MNN to form a nested mobile network. A nested mobile network of multiple levels is formed when an MR attaches to another MR. The MR which is directly attached to the wired network is called Top Level MR (TLMR) while MR1 is an MNN in TLMR's mobile network forming a nested mobile network. MRs attach to the Internet through Access Routers (ARs).

The network to which a mobile network is usually connected is called the home network. In home network, an MR is registered with a router called Home Agent (HA) that performs location tracking and packet re-direction for MNNs. In Fig. 1, HA-TLMR and HA-MR1 are the HA for the mobile routers TLMR and MR1, respectively. Also, a node that communicates with MNNs is termed as Correspondent Node $(\mathrm{CN})$.

\section{B. NEMO BSP}

An MR is assigned a prefix in its home network to advertise inside its mobile network. MNNs obtains addresses which is called home address from this prefix. Packets, sent to home address, reach the HA that forwards the packets to the mobile network which is attached to the home network. When a mobile network moves to a network (foreign network) other than home network, the MR obtains a new address called Careof-Address (CoA) from the prefix of the foreign network and

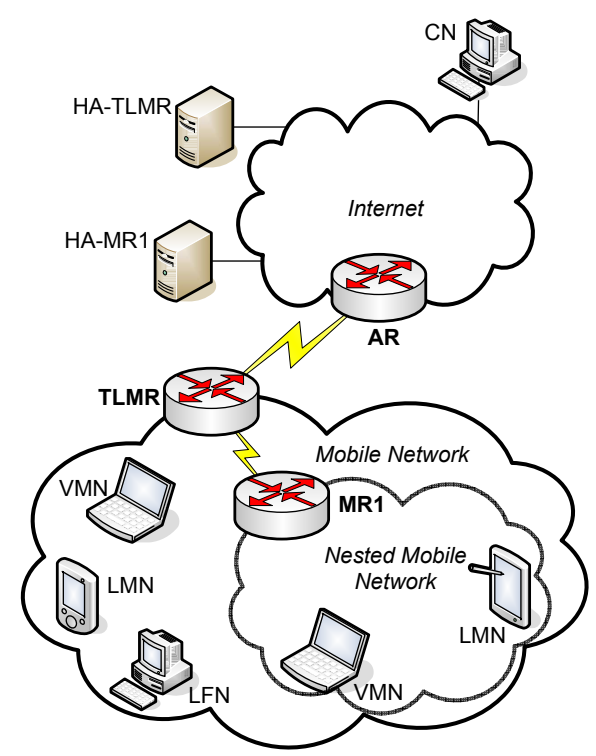

Fig. 1. Topology of a mobile network.

send a Binding Update (BU) to the HA informing the CoA. Therefore, HA intercepts packets sent to MNNs' home address and tunnels the packets to the CoA of the MR. Since a visiting mobile network which is nested under another mobile network gets CoA from the prefix (assigned at home network) of the visited mobile network, packets (sent to the visiting mobile network) will first go through HA of visiting mobile network and then through HA of visited mobile network. In case of multiple levels of nesting, packets have to go through all HAs of mobile networks up the nesting level resulting in suboptimal route and header overhead due to tunneling.

\section{PRefiX DELEGATION-BASED RoUte Optimization SCHEMES}

The basic idea behind prefix delegation-based route optimization is to let LMN, VMN and MR to obtain CoA from the prefix of the foreign network visited by the TLMR, and let the $\mathrm{CN}$ know the CoA. Therefore, $\mathrm{CN}$ can send packets to this CoA to have the packets directly (without going through HAs) reach the foreign network visited by TLMR. Different schemes apply the basic idea in different ways. Based on the differences (see Table I), we selected three schemes that represent all schemes in this class. Selected schemes and their differences are presented in the following subsections.

\section{A. Simple Prefix Delegation (SPD)}

In SPD, proposed by Lee et al. [7], MRs are delegated a prefix, that can be aggregated at TLMR's foreign network prefix, to advertise inside mobile network. MNNs (except LFNs) obtain CoA from the advertised prefix, and send the CoA to their respective HAs and CNs. This scheme defines a new neighbor discovery option, called Delegated Prefix Option, which is used by MRs to delegate prefix to attached MRs. Unlike other two schemes, presented in Sec. III, this scheme does not optimize LFNs' route, and consequently, 
TABLE I

DIFFERENCES AMONG THE THREE SCHEMES THAT MAKE DIFFERENCE IN THROUGHPUT.

\begin{tabular}{|l|l|l|}
\hline Schemes & CoA obtaining time & LFNs' route optimization \\
\hline SPD & Small & No \\
\hline MIRON & Large & Yes \\
\hline OPR & Small & Yes \\
\hline
\end{tabular}

packets sent to LFNs have to be tunneled through the HA of the LFNs' MR.

\section{B. Mobile IPv6-based Route Optimization (MIRON)}

In MIRON [8], an MR, after obtaining CoA, notifies (using PANA [10]) attached MNNs (except LFNs) to obtain CoA. MNNs send a DHCPv6 request which is relayed to the foreign network to which TLMR is attached. A DHCP server in the foreign network sends DHCPv6 reply (with a CoA configured from foreign network prefix) which is relayed down the nesting level to the MNNs. Unlike other two schemes, this $\mathrm{CoA}$ obtaining procedure takes longer time due to relay of request/reply upto the foreign network's DHCP server. Unlike SPD, route optimization for LFNs is done by MR send BUs to CNs on behalf of LFNs.

\section{Optimal Path Registration (OPR)}

In this scheme proposed by Park et al. [9], prefix delegation and $\mathrm{CoA}$ obtaining procedures are similar to that of SPD except only MRs obtain CoA from foreign network's prefix which is multi-cast to MRs through router advertisement. To optimize route for MNNs, MRs translate addresses of packets into new addresses by using delegated prefix and notify CNs about the new translated address by setting a bit in packets' header. Like SPD, OPR takes short time to obtain CoA whereas like MIRON, OPR optimizes route for LFNs.

\section{Performance critical differences among the schemes}

The schemes mainly differ in procedure of obtaining CoA and in optimizing route for LFNs. These differences, summarized in Table I, affect the throughput of the mobile network. MIRON takes longer time to obtain CoA resulting in longer handoff delay while MIRON and OPR optimize route for LFNs that results in shorter end-to-end delay. Handoff delay causes packet loss while throughput of protocols such as TCP is inversely proportional to end-to-end delay. Therefore, OPR will yield the highest throughput while throughput superiority of MIRON and SPD depends on mobility speed and end-toend delay between HA and mobile network. Rate of change of throughput with handoff frequency (determined by speed) is higher for MIRON due to high handoff delay. On the other hand, throughput of SPD changes with end-to-end delay between HA and mobile network while that of MIRON is unaffected. Evaluation of end-to-end delay, delay to obtain $\mathrm{CoA}$ and throughput under various speed and at different endto-end delay is presented in Sec. IV.

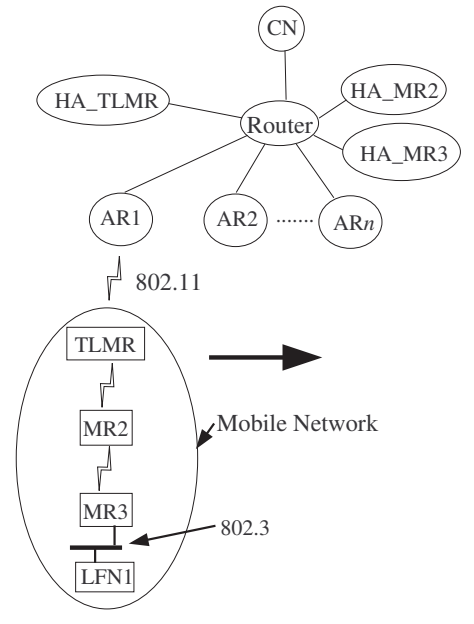

Fig. 2. Topology used for simulation.

TABLE II

VALUES OF PARAMETERS USED IN THE SIMULATION.

\begin{tabular}{|l|l|}
\hline Parameter & Value \\
\hline Simulation time & $360 \mathrm{~s}$ \\
\hline Wired link BW & $10 \mathrm{Mbps}$ \\
\hline Wired link delay & $1.8 \mathrm{~ms}$ \\
\hline Wireless (802.11b) link BW & $11 \mathrm{Mbps}$ \\
\hline Wireless range & $250 \mathrm{~m}$ \\
\hline Ethernet (802.3) BW & $10 \mathrm{Mbps}$ \\
\hline
\end{tabular}

\section{Performance evaluation}

To evaluate performance, we simulated the schemes, described in Sec. III, in ns-2 [11]. Simulation environment, analysis of the results and a comparative discussion are presented in the following subsections.

\section{A. Simulation Environment}

Fig. 2 demonstrates a topology used in simulation with two levels of nesting which was changed during experimentation. CN was the FTP source over TCP where as LFN1 (connected to MR3 using Ethernet) was the TCP sink. Mobile network moved between ARs, placed in a horizontal line. Speed of movement was varied between $2 \mathrm{~m} / \mathrm{s}$ to $30 \mathrm{~m} / \mathrm{s}$, and beyond $30 \mathrm{~m} / \mathrm{s}$ handoff is too frequent to be practical for real world scenario. Since throughput characteristics (with respect to speed) of all types of MNNs are the same across the schemes, and it is only LFNs' route optimization in which the schemes differ, we used only LFN in our evaluation. IEEE 802.11 standard was used wireless communications. To simulate change of end-toend delay between HA_MR3 and mobile network headed by $M R 3$, we varied the link delay between HA_MR3 and Router. Values of parameters, used in the simulation, are presented in Table II.

\section{B. Results}

We measured (at 95\% confidence level) end-to-end delay, delay to obtain CoA and throughput of the mobile network (residing at the lowest level) at different speed and at different end-to-end delay between the HA and the mobile network. 


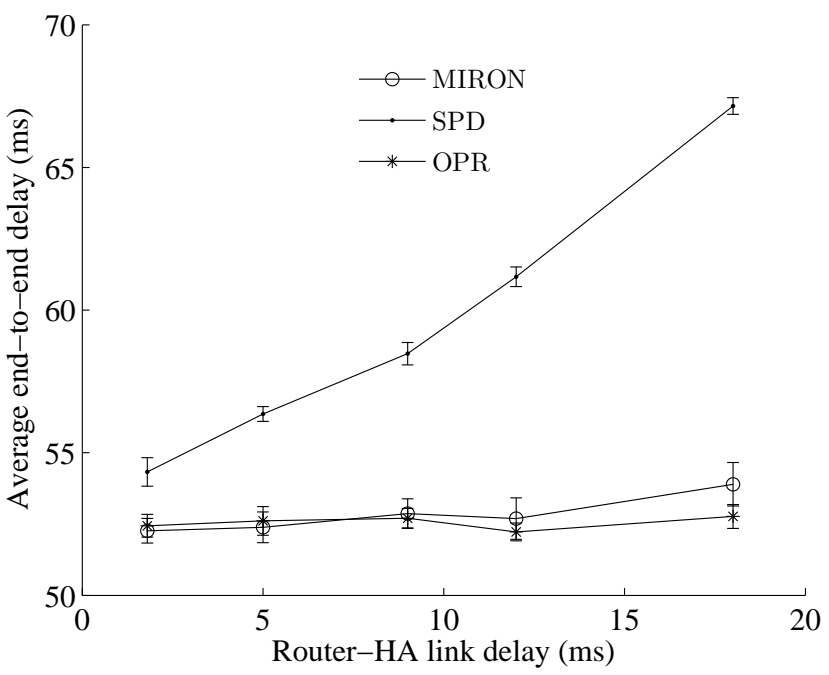

Fig. 3. Average end-to-end delay between CN and LFN1.

Throughput was measured by total amount of data received at LFN1. Analysis of results is as follows:

- End-to-end delay for LFNs: End-to-end delay, measured by the difference between the time of sending a packet by $\mathrm{CN}$ and the time of receiving the packet by LFN1, is shown in Fig. 3. With the increase of delay between Router and HA (in Fig. 2), end-to-end delay for SPD increases while that of the other two schemes is not affected much. The reason for this is described next. Since SPD does not optimize route, packets reach LFN1 through HA resulting in end-to-end delay of packets dependent on the end-to-end delay between Router and HA. Other two schemes optimize route for LFNs that enables packets to reach LFN1 without going through the HA, and consequently, end-to-end delay for these two schemes are not affected (except small increase in MIRON) by the end-to-end delay between Router and the HA.

In MIRON, end-to-end delay shows small increase because during handoff, $\mathrm{CN}$ may send packets through $\mathrm{HA}$ until a $\mathrm{BU}$ is received from MR. Sending of $\mathrm{BU}$ to $\mathrm{CN}$ might be delayed because at the reception of the first packet after handoff, MR marks the source $(\mathrm{CN})$ for sending BU (but does not send immediately) which is sent when the next period to send BU comes. During this delay, packets are sent through HA resulting in the increase of end-to-end delay in MIRON. In OPR, there is no delay because MR sends immediately CoA to $\mathrm{CN}$.

- Delay to obtain CoA vs. level of nesting We measured delay to obtain CoA for a nested MR (such as MR3 in Fig. 2) by measuring the difference between the time instant when TLMR obtains the CoA and the time instant when the nested MR obtains the CoA. This delay that affects the handoff delay is presented in Fig. 4.

For SPD and OPR, delays to obtain CoA are of similar order where each MR obtains CoA from the advertised prefix, and immediately advertises a part of the prefix to

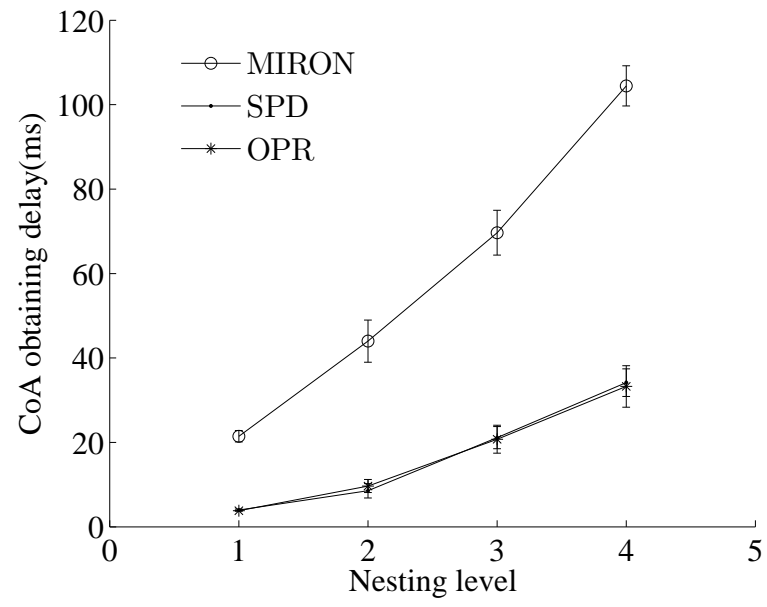

Fig. 4. CoA obtaining delay of the MR at the lowest level after TLMR hands off.

the attached MRs. All the MRs down the level does the same that results in a delay which is linearly proportional to the level.

For MIRON, this delay is much larger than that of SPD and OPR. An MR, after obtaining CoA, requests the attached MRs to obtain CoA using DHCPv6 request/reply that travels between the MRs and the TLMR's foreign network, and delay for this is proportional to the level of the MRs. Moreover, an MR can start procedure to obtain CoA after all the MRs above has obtained the CoA. Therefore, delay to obtain $\mathrm{CoA}$ is the sum of delay for all MRs above plus the delay for DHCPv6 request/reply, and quadratic in terms of level.

- Change of throughput with speed of mobile network: Fig. 5 shows the change of throughput with speed of the mobile network. Throughput falls with increasing speed due to increased handoff frequency. Since handoff delay of both SPD and OPR is lower due to low CoA obtaining time (see Fig. 4), and OPR optimizes route for LFNs, throughput for OPR is the highest. Despite having similar handoff delay, throughput of SPD is lower than OPR's throughput due to higher end-to-end delay (see Fig. 3). Although LFNs' route is optimized, rate of throughput fall with increasing speed is higher for MIRON because of high handoff delay due to higher delay to obtain CoA. Therefore, throughput of MIRON, higher at low speed, falls below throughput of SPD at higher speed because throughput fall due to handoff delay dominates throughput fall due to end-to-end delay at higher handoff frequency.

- Effect of level on fall of throughput: Another characteristic, observed from Fig. 5, is that fall of MIRON's throughput below SPD's throughput occurs at lower speed for higher level. The reason for this is the rate of increase of delay to obtain CoA (and hence, the rate of increase of handoff delay) with increase of level is higher for MIRON (see Fig. 4). Therefore, at higher level, rate of loss of throughput (due to handoff) of MIRON is higher 


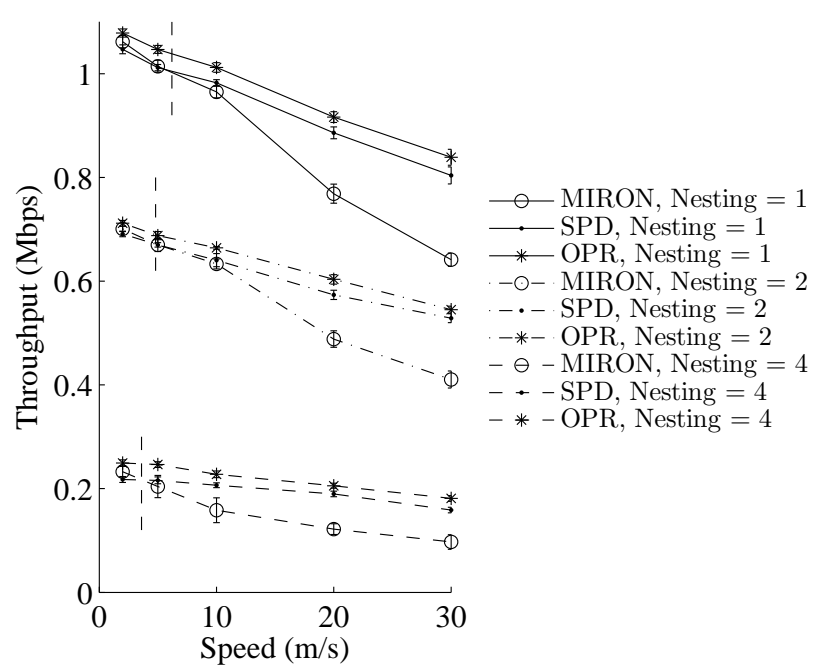

Fig. 5. Throughput of different schemes for different nesting level. Vertical dashed lines indicates the speed at which throughput of MIRON falls below 1... . . $\mathrm{cmn}$

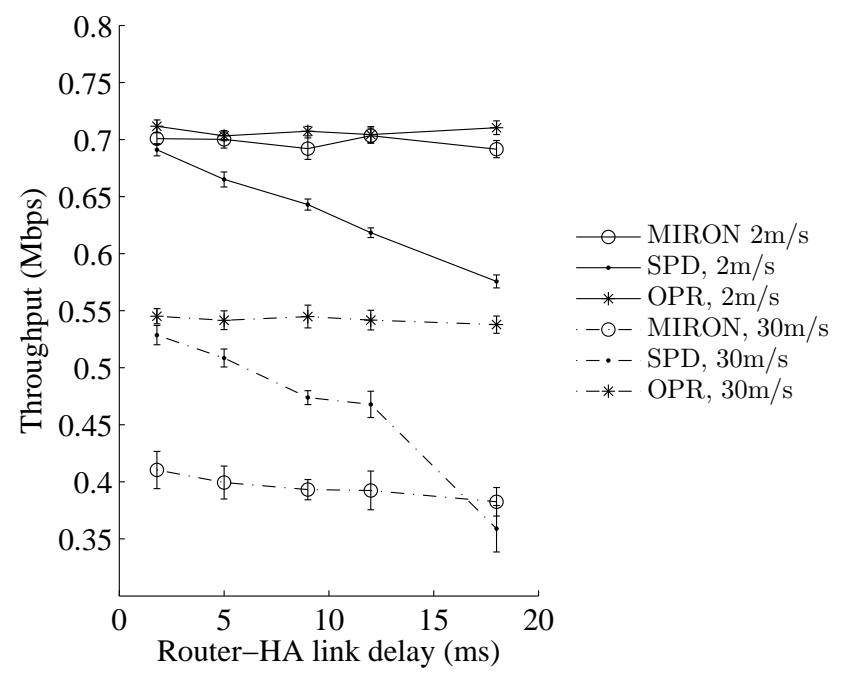

Fig. 6. Throughput of different schemes at two different speed.

than other schemes resulting in early fall (below SPD's throughput) of MIRON's throughput.

- Throughput vs. end-to-end delay between HA and mobile network: Fig. 6 shows the effect of end-to-end delay (between HA and mobile network) on throughput at two different speeds of mobile network. Since SPD does not optimize LFNs' route, packets go through HA. Therefore, increasing end-to-end delay between HA and mobile network results in decreasing throughput because TCP throughput is inversely proportional to delay. Endto-end delay for different schemes is shown in Fig. 3. Throughput of MIRON and OPR is not affected much by virtue of LFNs' route optimization that avoids packets' going through HA. Although MIRON's throughput is lower than SPD's throughput at higher speed, SPD's throughput falls below MIRON's throughput at higher end-to-end delay between HA and mobile network.
Based on the results presented in Sec. IV-B, we present a comparative discussion of the schemes in Sec. IV-C.

\section{Comparative discussion}

Although, from the results presented in Sec. IV-B, OPR appears to be the best, OPR needs packets to flow from mobile network to $\mathrm{CN}$. If packets are not flowing from mobile network to CN, OPR performs like SPD. MIRON always performs better than SPD at lower speed. Even at higher speed, MIRON performs better when mobile network is away from home network in terms of link delay. In addition, MIRON uses a feasible procedure for CoA obtaining. CoA obtaining procedure for both OPR and SPD by prefix delegation through router advertisement might not be easily applicable due to accounting and security reasons. Overall, OPR could be the best scheme if route optimization could be enabled when packets not flowing out from mobile network. MIRON is a good choice at low speed whereas SPD can be a good choice at high speed when mobile network stays close to home network.

\section{CONCLUSION}

In this paper, we evaluated the performance of prefix delegation-based schemes for NEMO route optimization. We simulated three schemes, namely MIRON, SPD and OPR, and measured end-to-end delay, delay to obtain CoA and throughput under various speeds at different end-to-end delays from the home network. Results show that OPR performs the best although OPR performance is limited due to inability to optimize route when packets are not sent from the mobile network to CN. MIRON, having a feasible solution for obtaining CoA, performs better at low speed. On the other hand, SPD can perform better at high speed when end-to-end delay between the home agent and the mobile network is small.

\section{REFERENCES}

[1] C. Perkins, "IP mobility support for IPv4," RFC 3220, Jan. 2002.

[2] D. B. Johnson, C. E. Parkins, and J. Arkko, "Mobility support in IPv6," RFC 3775, Jun. 2004.

[3] V. Devarapalli, R. Wakikawa, A. Petrescu, and P. Thubert, "NEtwork MObility (NEMO) basic support protocol," RFC 3963, Jan. 2005.

[4] M. Watari, T. Ernst, and J. Murai, "Routing optimization for nested mobile networks," IEICE Transaction on Communication, vol. E89-B, no. 10 , pp. 2786-2793, Oct. 2006.

[5] C. Ng, F. Zhao, M. Watari, and P. Thubert, "Network mobility route optimization solution space analysis," RFC 4889, Jul. 2007.

[6] H. Lim, D. Lee, T. Kim, and T. Chung, "A model and evaluation of route optimization in nested NEMO environment," IEICE Transaction on Communication, vol. E88-B, no. 7, pp. 2765-2776, Jul. 2005.

[7] K. Lee, J. Park, and H. Kim, "Route optimization for mobile nodes in mobile network based on prefix delegation," in IEEE 58th Vehicular Technology Conference, Orlando, Florida, USA, Oct. 6-9, 2003, pp. 2035-2038.

[8] M. Calderon, C. J. Bernardos, M. Bagnulo, I. Soto, and A. de la Oliva, "Design and experimental evaluation of a route optimization solution for NEMO," IEEE Journal on Selected Areas in Communications, vol. 24, no. 9, pp. 1702-1716, Sep. 2006.

[9] H. Park, T. Lee, and H. Choo, "Optimized path registration with prefix delegation in nested mobile networks," in International Conference on Mobile Ad-hoc and Sensor Networks, Wuhan, China, Dec. 13-15, 2005.

[10] D. Forsberg, Y. Ohba, B. Patil, H. Tschofenig, and A. Yegin, "Protocol for carrying authentication for network access (PANA)," RFC 5191, May 2008.

[11] “The network simulator - ns-2," http://www.isi.edu/nsnam/ns/. 